\title{
The equine DNAH3 gene: SNP discovery and exclusion of an involvement in recurrent airway obstruction (RAO) in European Warmblood horses
}

\author{
Mostafa Shakhsi-Niaei', Jolanta Klukowska-Rötzler ${ }^{1}$, Cord Drögemüller ${ }^{1}$, Vincent \\ Gerber $^{2}$ and Tosso Leeb ${ }^{1}$ \\ ${ }^{1}$ Institute of Genetics, Vetsuisse Faculty, University of Bern, Bern, Switzerland, ${ }^{2}$ Swiss Institute of Equine Medicine, \\ Vetsuisse Faculty, University of Bern and ALP-Haras, Bern, Switzerland
}

\section{Abstract}

Recurrent airway obstruction is one of the most common airway diseases affecting mature horses. Increased bronchoalveolar mucus, neutrophil accumulation in airways, and airway obstruction are the main features of this disease. Mucociliary clearance is a key component of pulmonary defense mechanisms. Cilia are the motile part of this system and a complex linear array of dynein motors is responsible for their motility by moving along the microtubules in the axonemes of cilia and flagella. We previously detected a QTL for RAO on ECA 13 in a half-sib family of European Warmblood horses. The gene encoding DNAH3 is located in the peak of the detected QTL and encodes a dynein subunit. Therefore, we analysed this gene as a positional and functional candidate gene for RAO. In a mutation analysis of all 62 exons we detected 53 new polymorphisms including 7 non-synonymous variants. We performed an association study using 38 polymorphisms in a cohort of 422 animals. However, after correction for multiple testing we did not detect a significant association of any of these polymorphisms with RAO $(P>0.05)$. Therefore, it seems unlikely that variants at the DNAH3 gene are responsible for the RAO QTL in European Warmblood horses.

Keywords: horse; allergy; recurrent airway obstruction; dynein; association

Abbreviations: ATP: adenosine-5'-triphosphate; DNAH: dynein heavy chain; ECA: equine chromosome; HOARSI: horse owner assessed respiratory signs index; PCD: primary ciliary dyskinesia; QTL: quantitative trait locus; RAO: recurrent airway obstruction; SNP: single nucleotide polymorphism; $\mathrm{T}_{\mathrm{m}}$ : melting temperature 


\section{Introduction}

RAO or heaves is one of the most common airway diseases affecting mature horses. RAO is characterised by bronchospasms and increased mucus and neutrophil accumulation in the airways. It occurs following exposure of susceptible horses to antigens and endotoxins present in hay and stable dust (Cunningham \& Dunkel 2008, Léguillette 2003). Because of many similarities of this disease to human asthma it is used as an animal model of human asthma (Deaton 2006, Leclere et al. 2011).

The prevalence of RAO is estimated at $10-20 \%$ of adult horses living in cold and temperate climates. There is no breed or sex predisposition for RAO (Leclere et al. 2011). RAO is a complex trait controlled by environmental and genetic factors. In a Warmblood population there was evidence for the presence of one or more major genes and the heritability in a study cohort exposed to hay feeding was estimated to approach $100 \%$. Thus hay-feeding may be the only significant environmental factor influencing this disease (Gerber et al. 2009).

Mucociliary clearance is a key component of pulmonary defense mechanisms. It involves the regulation of ion transport by the airway epithelium, mucus secretion, and ciliary function (Knowles \& Boucher 2002). Cilia and flagella contain motile microtubules with a "9+2«structure, in which two central singlet microtubules are surrounded by nine outer doublet microtubules. A complex linear array of dynein motors is responsible for their motility (Brokaw 2009, Hayashi \& Shingyogi 2008).

Cytoplasmic and axonemal dyneins are involved in the cytoplasmatic movement of organelles and the bending of cilia and flagella, respectively (Bartoloni et al. 2001). The DNAH proteins assemble with intermediate and light chains into large multiprotein complexes to form inner and outer dynein arms (Holzbaur \& Vallee 1994, DiBella \& King 2001). The inner and outer dynein arms slide on the outer doublet microtubules by hydrolysing ATP.

Defects in the motile cilia are responsible for the most prominent ciliopathy in mammals, called primary ciliary dyskinesia. One aspect of PCD is respiratory disease due to impaired mucociliary clearance. Additional features of PCD are sperm immobility and randomisation of left-right asymmetry (Afzelius 1976, Rossman et al. 1980). The different features of PCD are now clearly explained by mutations involving various subunits of the axonemal structures (Lee 2011).

Due to their importance for mucociliary clearance the genes encoding dynein subunits can be considered functional candidates for RAO. In a previous microsatellite-based linkage study we detected a QTL for RAO in a half-sibling family of European Warmblood horses with a peak of about $4 \mathrm{Mb}$ extending from 24-28 Mb on ECA 13 (Swinburne et al. 2009). The DNAH3 gene expressed in trachea, testis and brain (Maiti et al. 2000) is located on ECA 13 at 26.4$26.6 \mathrm{Mb}$ in the peak region of the detected QTL. Therefore, we analysed the equine DNAH3 gene as a positional and functional candidate gene and performed an association study in a cohort of European Warmblood horses. 


\section{Material and methods}

\section{Animals and phenotypes}

For variant detection we selected two RAO-affected and two non-affected half-sibling offspring of a previously described European Warmblood family, in which the QTL on ECA 13 was detected (Swinburne et al. 2009). We selected these four horses based on their phenotypes and marker haplotypes at the QTL.

For the association analysis we used 464 European Warmblood horses that were unrelated at the grandparental level. More than $60 \%$ (281) of the sampled horses were from Switzerland and Germany. Additional horses were from Belgium, the Czech Republic, Denmark, France, Hungary, Ireland, Latvia, The Netherlands, Poland, Portugal, Russia, Slovakia, and Sweden. Cases and controls were matched according to countries in order to minimise the stratification. A multidimensional scaling plot of genome-wide SNP data from these horses did not result in different clusters between cases and controls (data not shown).

We determined the phenotypes in our sample population according to the "Horse Owner Assessed Respiratory Signs Index“ (Ramseyer et al. 2007, Laumen et al. 2010). The HOARSI gives scores from 1-4, which correspond to absent, mild, moderate and severe clinical signs respectively. Briefly, horse owners were contacted by phone and only horses with clinical signs that had persisted for at least 2 months were included in the study. All horses were 5 years or older, with at least a 12-month history of hay-feeding. A standardised questionnaire was used to gather information from the horse owners about the animals' history of chronic, regular or frequent coughing, increased breathing effort at rest, increased breathing effort after exercise, and nasal discharge. The HOARSI classification refers to the period when the horses were exhibiting the most severe clinical signs. We selected horses with HOARSI 1 as controls and horses with HOARSI 3 or 4 as RAO cases. We used an overlapping set of horses in a related study (Shakhsi-Niaei et al. 2012).

\section{Mutation analysis and genotyping}

We amplified all 62 DNAH3 exons and their flanking regions in 4 horses (Table 1). The PCR products were directly sequenced on an ABI 3730 sequencer (LifeTechnologies, Carlsbad, CA, USA) and polymorphisms were identified using Sequencher software v4.9. (GeneCodes, Ann Arbor, MI, USA). The newly identified variants were submitted to the Single Nucleotide Polymorphism Database (dbSNP, http://www.ncbi.nlm.nih.gov/projects/SNP/).

For the association study we determined the genotypes of 35 SNPs by using Golden Gate assays on a BeadXpress station (Illumina Inc., San Diego, CA, USA) followed by data analysis with the BeadStudio v3 software (Illumina Inc, San Diego, CA, USA). The genotypes of 4 additional SNPs in the region were available from equine SNP50 Beadchip data (Illumina Inc, San Diego, CA, USA). All genomic positions refer to the EquCab 2 assembly (http://www. ncbi.nlm.nih.gov/projects/mapview/). The equine cDNA and protein positions refer to the database accessions XM_001491853.3 and XP_001491903.2, respectively. 
Table 1

Primer sequences. The positions correspond to chromsome 13 in the Equus caballus 2 genome assembly.

\begin{tabular}{|c|c|c|c|c|c|c|c|c|}
\hline & Start & Stop & Exon & Primer & Forward Sequence $\left(5^{\prime}>3^{\prime}\right)$ & Reverse Sequence $\left(5^{\prime}>3^{\prime}\right)$ & $\mathrm{T}_{\mathrm{m}}$ & Product, bp \\
\hline 1 & 26.419 .946 & 26.420 .804 & 1 & DNAH3_P1 & TGCATTTAGGCCTCTCCAA & ССТTСАСАСССАСТTCCAА & 59.43 & 859 \\
\hline 2 & 26.424 .250 & 26.425 .322 & $2+3$ & DNAH3_P2+3 & TGGGGATGAGAAGGGTGTT & ACAGCCAGGACCAGAATCC & 55.16 & 1073 \\
\hline 3 & 26.427 .888 & 26.428 .853 & $4+5$ & DNAH3_P4+5 & TCCCCTTAGTGTGCTCCTG & GCTGCCATTCTGCAACCTA & 59.39 & 966 \\
\hline 4 & 26.431 .430 & 26.432 .444 & 6 & DNAH3_P6 & TCTGGTGTCATGGGGAAAA & TGACCTACTGCGTGCACAA & 59.96 & 1015 \\
\hline 5 & 26.432 .363 & 26.433 .609 & 7 & DNAH3_P7 & AGCTCTTTGATGGTGATGGA & GCAGGGCTACTCCCACAGT & 59.14 & 1247 \\
\hline 6 & 26.436 .353 & 26.437 .412 & 8 & DNAH3_P8 & AGCCATCAGGGGTAGGTCT & GGATTTCTCTGGCTTTGGA & 58.17 & 1060 \\
\hline 7 & 26.438 .081 & 26.438 .589 & 9 & DNAH3_P9 & ATGAGCCATCTGCCTTGTC & AACGACTTCTCAGCTTCTGC & 58.09 & 509 \\
\hline 8 & 26.439 .899 & 26.440 .415 & 10 & DNAH3_P10 & AGCCATACCCAGCCCTTAT & AGATGCACGGCCATATTTT & 58.27 & 517 \\
\hline 9 & 26.440 .671 & 26.441 .693 & 11 & DNAH3_P11 & GCCCAAGCTCTTTCCAGT & TCATGCTGACGTTTTTCCA & 58.09 & 1023 \\
\hline 10 & 26.442 .941 & 26.443 .713 & 12 & DNAH3_P12 & GGAAGTGGGGAGAGGAAGT & TGCTGCCTTTTCTTATCATGT & 57.81 & 773 \\
\hline 11 & 26.445 .159 & 26.446 .002 & $13+14$ & DNAH3_P13+14 & TTCAGTGTATGTTTTGCCACA & ATTGCCTCAGGATGCTTTC & 57.75 & 844 \\
\hline 12 & 26.450 .454 & 26.451 .432 & 15 & DNAH3_P15 & CCCATCAAGGATTTTTCAGG & GGTAGAGGGCACAGCTCAT & 57.87 & 979 \\
\hline 13 & 26.451 .267 & 26.452 .213 & 16 & DNAH3_P16 & GCTCTCCACAAGCCAAGTT & CAGTGAAGCAGGCTGAAAA & 57.85 & 947 \\
\hline 14 & 26.460 .129 & 26.460 .774 & 17 & DNAH3_P17 & AATGTGACTGGGCTTTCGT & GCTGACTTGCCTCAAAGGT & 58.08 & 646 \\
\hline 15 & 26.460 .362 & 26.461 .431 & 18 & DNAH3_P18 & TTGGTTCTGGTCCTTGGTT & TCСССАСТTТССАТССТТА & 57.94 & 1070 \\
\hline 16 & 26.470 .588 & 26.471 .110 & 19 & DNAH3_P19 & GCTCAGGGAATTACAGCTACA & CTGGCTGGCACAATACTCA & 57.76 & 523 \\
\hline 17 & 26.474 .688 & 26.475 .249 & 20 & DNAH3_P20 & CAAGTGCTGGTGGGAGAC & CACCCAAAGTGAAGGTTCC & 57.79 & 562 \\
\hline 18 & 26.478 .447 & 26.478 .714 & 21 & DNAH3_P21 & TGTCTCCTCCCGTTGAGTT & GGGACCCTTTCTCTGTGAG & 57.69 & 268 \\
\hline 19 & 26.485 .549 & 26.485 .919 & 22 & DNAH3_P22 & GGGTGGAGATCTGAGCTGT & TGCCCTTACTTGGATGGTT & 57.86 & 371 \\
\hline 20 & 26.486 .494 & 26.486 .969 & 23 & DNAH3_P23 & ATCAGTGGGGCTGTGATG & CCTCTAGGGAGGTGGGATT & 57.93 & 476 \\
\hline 21 & 26.487 .920 & 26.488 .405 & 24 & DNAH3_P24 & TGGCTCAGTTGGTTTCAGA & GTCTCTTGGGGAATGGAGA & 57.71 & 486 \\
\hline 22 & 26.491 .118 & 26.491 .979 & 25 & DNAH3_P25 & CAACCTCTATGCCCCAGAC & CGGCAGGAGTGGAAGTAAT & 57.92 & 862 \\
\hline 23 & 26.492 .393 & 26.492 .909 & 26 & DNAH3_P26 & TGTCATTTGCCTCCTGAGA & GCCCCAAGATTTACAAGGA & 57.70 & 517 \\
\hline 24 & 26.494 .125 & 26.494 .769 & 27 & DNAH3_P27 & TAAGCCCAGACGTGTCAAG & ATTGGAGGAGGAGGAGAGG & 57.79 & 645 \\
\hline 25 & 26.497 .108 & 26.498 .098 & 28 & DNAH3_P28 & GCTAATGCCTGGAGGTCAG & GGGTGTGTGAGGAGGAAAG & 58.22 & 991 \\
\hline 26 & 26.499 .486 & 26.499 .957 & 29 & DNAH3_P29 & AGTGAGAGGTGGCCTTCTG & AGTAAGAGCCCTGCTTGGA & 58.01 & 506 \\
\hline 27 & 26.501 .026 & 26.501 .613 & $30+31$ & DNAH3_P30+31 & CCAGTTTCTTTCCCGTGTC & CCTGTCATTGGGGAGCTG & 59.16 & 588 \\
\hline 28 & 26.501 .601 & 26.502 .619 & 32 & DNAH3_P32 & CCCCAATGACAGGGTCTTA & TGTTGGGAAAGTGGTTGTG & 57.67 & 1019 \\
\hline 29 & 26.503 .380 & 26.504 .117 & 33 & DNAH3_P33 & AGCCCTCCTTTCCTTGATT & ACTCTTGGTGCGTGTGATG & 57.99 & 738 \\
\hline 30 & 26.505 .289 & 26.506 .431 & $34+35$ & DNAH3_P34+35 & CAAATGATAGGGCTTCTCCA & TTCCTGACTCCAGCAAACA & 57.43 & 1143 \\
\hline 31 & 26.507 .364 & 26.507 .813 & 36 & DNAH3_P36 & TAGTTGGGTGCAGAGCAAA & AGAATCCAGGGACAGCAAA & 58.10 & 450 \\
\hline
\end{tabular}


Table 1 continued

Primer sequences. The positions correspond to chromsome 13 in the Equus caballus 2 genome assembly.

\begin{tabular}{|c|c|c|c|c|c|c|c|c|}
\hline & Start & Stop & Exon & Primer & Forward Sequence $\left(5^{\prime}>3^{\prime}\right)$ & Reverse Sequence $\left(5^{\prime}>3^{\prime}\right)$ & $T_{m}$ & Product, bp \\
\hline 32 & 26.509 .133 & 26.509 .688 & 37 & DNAH3_P37 & TGTTGGCTGGTCTTTGAGA & AGCAACAAGGCTTTTAACCA & 57.72 & 556 \\
\hline 33 & 26.512 .163 & 26.512 .417 & 38 & DNAH3_P38 & TCAGACCATCTCTGTCTCCAC & GAGCTGTCCCTCCTGCTTA & 57.94 & 255 \\
\hline 34 & 26.514 .273 & 26.514 .847 & 39 & DNAH3_P39 & CTGGTCCTGGAGTTGGTGT & GTGAGGCAATGGAGAAAGG & 58.36 & 575 \\
\hline 35 & 26.516 .444 & 26.516 .835 & 40 & DNAH3_P40 & CCTATGGGCCGTACAATTC & TCATGGGAGGAAAAACAGG & 58.19 & 392 \\
\hline 36 & 26.519 .054 & 26.519 .787 & 41 & DNAH3_P41 & GGCCTCTCACAACAAAACC & CAAACGGCTTAGGGACATT & 57.90 & 734 \\
\hline 37 & 26.531 .911 & 26.532 .668 & 42 & DNAH3_P42 & CTGACAACATGGAGTTTCACC & AATGGGCAGGAGTTGTAGG & 57.82 & 758 \\
\hline 38 & 26.534 .404 & 26.535 .138 & 43 & DNAH3_P43 & TGCCAAGACATAGCCTCCT & ACACCACAATCCCCTTCTG & 58.37 & 735 \\
\hline 39 & 26.536 .286 & 26.536 .965 & 44 & DNAH3_P44 & TGCCAATCTGTGCATCTTT & TGGAGGCTTTCTGTTCCTC & 57.85 & 680 \\
\hline 40 & 26.541 .797 & 26.542 .677 & $45+46+47$ & DNAH3_P45+46+47 & GGATAACTAGGCCCAAGAGG & AACGGAACCCGAAATCTG & 58.13 & 881 \\
\hline 41 & 26.543 .437 & 26.544 .439 & 48 & DNAH3_P48 & GAATGTGCTCAGGGGAAAC & TGGAGAGGGTTCAGAGGAA & 58.17 & 1003 \\
\hline 42 & 26.545 .331 & 26.545 .711 & 49 & DNAH3_P49 & CACTTCCCAGGCACTCAC & TGGAATATTTGGTTGGGGTA & 57.64 & 381 \\
\hline 43 & 26.547 .068 & 26.547 .557 & 50 & DNAH3_P50 & САCCTCCCCAGTTCTTCTC & GTCTCATTTGGTGCCCTCT & 57.40 & 490 \\
\hline 44 & 26.551 .417 & 26.551 .713 & 51 & DNAH3_P51 & TCATTTCTGCTCTTGCCAAT & GTTCCCTCCCTTTGCTCTC & 58.63 & 297 \\
\hline 45 & 26.556 .761 & 26.557.283 & 52 & DNAH3_P52 & CCAGTTAGAGCCACCCTTG & ACATCCTGGTGCCATTCTT & 58.11 & 523 \\
\hline 46 & 26.560 .227 & 26.561 .280 & 53 & DNAH3_P53.1 & GGCTTTACGTCTCGACTGG & TGCTGCTTGAATGTGGACT & 58.18 & 1054 \\
\hline 47 & 26.559 .366 & 26.560 .614 & 53 & DNAH3_P53.2 & GCTCCTGTTGCCTTCTCC & TCTGCTCGCTTCTGGTTC & 58.06 & 1249 \\
\hline 48 & 26.560 .466 & 26.561 .379 & 53 & DNAH3_P53.3 & TCTGCCTGTGAGGGTCTGT & GGGAGGTAATGTGGGTTCC & 59.22 & 914 \\
\hline 49 & 26.561 .162 & 26.562.138 & 53 & DNAH3_P53.4 & TATGTGAGGACGCTGGAAA & ATCAGGCCTTCGAGTTTTG & 57.82 & 977 \\
\hline 50 & 26.561 .940 & 26.562 .481 & 53 & DNAH3_P53.5 & AGCTGCTTTTTCTCCCTCCT & TCCACCCATTCTGGACTTT & 58.06 & 542 \\
\hline 51 & 26.566 .835 & 26.567 .681 & 54 & DNAH3_P54 & TCAGAGCAAAAGAGGAATCAA & TCACCTCCCTCCAACAACT & 57.87 & 847 \\
\hline 52 & 26.570 .239 & 26.570 .543 & 55 & DNAH3_P55 & GGGTCTGTTGTTCCTCCAG & GATCTCTCCCCCTGCAAC & 58.05 & 305 \\
\hline 53 & 26.571 .932 & 26.572 .318 & 56 & DNAH3_P56 & ATTCATGGGGCTGAAAGAG & CCAGCACAGTAACCAGCAT & 57.46 & 387 \\
\hline 54 & 26.575 .939 & 26.576.594 & 57 & DNAH3_P57 & GTAGAGCTCCTTGGCTGGA & ССССТTTTTCATCCTTCCT & 58.07 & 656 \\
\hline 55 & 26.576.497 & 26.577.249 & 58 & DNAH3_P58 & TTCAGAAGGTGCCAGAAGG & TGGAAAACTGCATGGAAAA & 58.30 & 753 \\
\hline 56 & 26.578 .965 & 26.579 .435 & 59 & DNAH3_P59 & TTCACTGCTTGAAGTTTACCC & TACGGTTAGCCTGTGCTTG & 57.27 & 471 \\
\hline 57 & 26.579 .711 & 26.580 .619 & 60 & DNAH3_P60 & ATCTGGAGCAGCAAGAACC & TCCCCTAACTCTCGGAAGA & 57.67 & 909 \\
\hline 58 & 26.581 .215 & 26.582 .035 & 61 & DNAH3_P61 & CAGCTTGTACCTGGGCTTT & TTGGTATGATGGTGGCAGA & 58.19 & 821 \\
\hline 59 & 26.582.915 & 26.583 .900 & 62 & DNAH3_P62 & GCATCTGACTCTAACCCCACT & ACCCAAACCTCTTTCAACCT & 57.94 & 986 \\
\hline
\end{tabular}




\section{Protein analysis}

In order to analyse the evolutionary conservation of the non-synonymous variants, we prepared multi-species alignments of the DNAH3 protein sequences using the ClustalW2 software (European Bioinformatics Institute, Cambridge, UK; http://www.ebi.ac.uk/Tools/ msa/clustalw2/).

\section{Association analysis}

We removed individuals and markers with less than $90 \%$ call rate with the Plink software (Purcell et al. 2007). We also excluded markers with a minor allele frequency of less than 0.05 and markers that strongly deviated from Hardy-Weinberg equilibrium $(P<0.001)$. Subsequently, we performed an allelic association study using chi-square tests. We also performed haplotypic association analyses using sliding windows of 3 and 7 markers, respectively. We corrected for multiple testing by applying the Bonferroni correction.

\section{Results and discussion}

\section{Mutation analysis of the DNAH3 gene}

We sequenced 34156 nucleotides containing all 62 exons with 12255 nucleotides of coding sequence of the DNAH3 gene in two RAO affected and two control horses. These four horses were half-sibling offspring of the European Warmblood family, in which the QTL on ECA 13 was originally detected. We identified a total of 53 new polymorphisms including 7 nonsynonymous variants in addition to 4 previously known SNPs in the region (Table 2). Protein sequence alignment showed that 4 of these 7 non-synonymous variants led to amino acid exchanges in highly conserved positions (p.Gln1227Arg, p.Met2357Val, p.GIn2665Arg, p.Ser3033Cys; Figure 1). These variants are located either in or very close to functionally annotated domains of the DNAH3 protein (Table 3). It has to be cautioned that the 5 '-end of the predicted horse DNAH3 transcript (XM_001491853.3) corresponding to the first exon is very different from the human ortholog. Thus there may be an error in the equine genome assembly or the annotation of the equine DNAH3 gene. Such a hypothetical error might alter the positions of the identified variants by about 30 amino acids. The human-horse relationships are given in Table 3.

\section{Association analysis of DNAH3 variants with RAO}

For the association study we genotyped 39 SNPs including all 13 exonic variants in a cohort of 464 animals. We removed one marker due to low minor allele frequency and 42 horses due to low call rates, so that 422 horses ( 230 cases \& 192 controls) and 38 markers remained for the final allelic association analysis (Table 2). All of the tested markers were in Hardy-Weinberg equilibrium and had similar allele distributions between cases and controls with raw $P$-values greater than $5 \%$. As 38 markers were tested, a Bonferroni-corrected significance threshold would have been $0.05 / 38=0.0013$. We also did not detect a significant haplotype association. 
Table 2

Polymorphisms of the equine DNAH3 gene and association data

\begin{tabular}{|c|c|c|c|c|c|c|c|c|c|c|}
\hline SNP & $\begin{array}{l}\text { DNAH3 } \\
\text { Position }\end{array}$ & $\begin{array}{l}\text { ECA } 13 \\
\text { Position }\end{array}$ & Function & $\begin{array}{c}\text { Allele } \\
1\end{array}$ & $\begin{array}{c}\text { Frequenc) } \\
\text { Cases } \\
(\mathrm{n}=230)\end{array}$ & $\begin{array}{l}\text { y Allele } 1 \\
\text { Controls } \\
(n=192)\end{array}$ & $\begin{array}{l}\text { Allele } \\
2\end{array}$ & $\begin{array}{l}\text { Chi- } \\
\text { square }\end{array}$ & $P$-value & $\begin{array}{l}\text { Odds } \\
\text { Ratio }\end{array}$ \\
\hline c. $-263 C>T$ & $5^{\prime}$-UTR & 26420431 & - & C & 0.400 & 0.466 & $\mathrm{~T}$ & 3.706 & 0.054 & 0.764 \\
\hline c. $-206 \mathrm{~T}>\mathrm{A}$ & $5^{\prime}$-UTR & 26420488 & - & $\mathrm{T}$ & 0.446 & 0.396 & A & 2.169 & 0.141 & 1.230 \\
\hline c. $135+46 C>G$ & intron 2 & 26424538 & - & & - & - & & - & - & - \\
\hline c. $135+238 \mathrm{~T}>\mathrm{C}$ & intron 2 & 26424730 & - & C & 0.469 & 0.486 & $\mathrm{~T}$ & 0.212 & 0.645 & 0.936 \\
\hline c. $135+336 C>T$ & intron 2 & 26424828 & - & & - & - & & - & - & - \\
\hline c. $135+388 \mathrm{~T}>\mathrm{C}$ & intron 2 & 26424880 & - & & - & - & & - & - & - \\
\hline c. $243 \mathrm{~T}>\mathrm{C}$ & exon 3 & 26425126 & $\mathrm{p} .=$ & $\mathrm{T}$ & 0.074 & 0.091 & C & 0.828 & 0.363 & 0.796 \\
\hline c. $431+11 A>G$ & intron 4 & 26428077 & - & G & 0.465 & 0.479 & A & 0.167 & 0.683 & 0.945 \\
\hline c. $431+169 C>A$ & intron 4 & 26428235 & - & & - & - & & - & - & - \\
\hline BIEC2-226005 & intron 5 & 26429354 & - & C & 0.454 & 0.466 & $\mathrm{~T}$ & 0.129 & 0.720 & 0.951 \\
\hline BIEC2-226006 & intron 5 & 26429577 & - & $\mathrm{T}$ & 0.454 & 166 & G & 0.129 & 0.720 & 0.951 \\
\hline c. $992+3309 A>T$ & intron 7 & 26436824 & - & A & 0.450 & 0.477 & $\mathrm{~T}$ & 0.594 & 0.441 & 0.899 \\
\hline c. $1033 \mathrm{~A}>\mathrm{G}$ & exon 8 & 26436881 & p.Lys345Glu & G & 0.352 & 0.397 & A & 1.787 & 0.181 & 0.825 \\
\hline c. $1494+58 A>G$ & intron 10 & 26440284 & - & & - & - & & - & - & - \\
\hline c. $14942+936 C>T$ & intron 10 & 26441163 & - & $\mathrm{T}$ & 0.448 & 0.474 & C & 0.569 & 0.451 & 0.900 \\
\hline c. $1531 G>A$ & exon 11 & 26441265 & p.Val511lle & A & 0.450 & 0.477 & G & 0.603 & 0.438 & 0.898 \\
\hline c. $1824+121 C>T$ & intron 13 & 26445527 & - & C & 0.441 & 0.448 & $\mathrm{~T}$ & 0.037 & 0.847 & 0.974 \\
\hline c. $2424+558 \mathrm{~A}>\mathrm{G}$ & intron 17 & 26460868 & - & G & 0.091 & 0.096 & A & 0.063 & 0.802 & 0.942 \\
\hline c. $2781+168 \mathrm{~T}>\mathrm{C}$ & intron 19 & 26471046 & - & & - & - & & - & - & - \\
\hline c. $2988+12 T>G$ & intron 21 & 26478616 & - & $\mathrm{T}$ & 0.067 & 0.073 & G & 0.098 & 0.754 & 0.919 \\
\hline c. $3285+49 \mathrm{~T}>C$ & intron 23 & 26486786 & - & $\mathrm{T}$ & 0.194 & 0.181 & C & 0.226 & 0.635 & 1.088 \\
\hline c. $3624+52 \mathrm{G}>\mathrm{A}$ & intron 25 & 26491543 & - & & - & - & & - & - & - \\
\hline c. $3624+85 C>T$ & intron 25 & 26491576 & - & & - & - & & - & - & - \\
\hline c. $3624+124 T>C$ & intron 25 & 26491615 & - & & - & - & & - & - & - \\
\hline c. $3624+272 \mathrm{G}>\mathrm{A}$ & intron 25 & 26491763 & - & & - & - & & - & - & - \\
\hline c. $3624+306 \mathrm{~T}>\mathrm{C}$ & intron 25 & 26491797 & - & & - & - & & - & - & - \\
\hline c. $3624+1110 G>A$ & intron 25 & 26492602 & - & A & 0.363 & 0.375 & G & 0.127 & 0.721 & 0.950 \\
\hline c. $3680 A>G$ & exon 26 & 26492718 & p.GIn1227Arg & A & 0.312 & 0.307 & G & 0.024 & 0.877 & 1.023 \\
\hline c. $4142+60 T>C$ & intron 29 & 26499841 & - & $\mathrm{T}$ & 0.480 & 0.453 & C & 0.627 & 0.429 & 1.116 \\
\hline c. $4272 A>G$ & exon 31 & 26501439 & $p .=$ & A & 0.435 & 0.430 & G & 0.022 & 0.882 & 1.021 \\
\hline c. $4634+314 A>G$ & intron 33 & 26503913 & - & & - & - & & - & - & - \\
\hline c. $4815 C>T$ & & 26505595 & $\mathrm{p} .=$ & C & 0111 & 0.079 & $\mathrm{~T}$ & 3.297 & 0.069 & 1.542 \\
\hline c. $4854+525 \mathrm{~A}>\mathrm{T}$ & intron 34 & 26506159 & - & $T$ & & 3 & A & 2.653 & 0.103 & 1.463 \\
\hline c. $4989+1139 T>C$ & intron 35 & 26507511 & - & $\mathrm{T}$ & 0.298 & 0.302 & C & 0.018 & 0.893 & 0.980 \\
\hline c. $5101+1509 C>T$ & intron 36 & 26509216 & - & $\mathrm{T}$ & 0.137 & 0.104 & c & 2.100 & 0.147 & 1.365 \\
\hline c. $5160 \mathrm{~T}>\mathrm{C}$ & exon 37 & 26509281 & $\mathrm{p} .=$ & $\mathrm{T}$ & 0.117 & 0.081 & C & 3.020 & 0.082 & 1.506 \\
\hline c. $5343+22 A>G$ & intron 37 & 26509486 & - & - & - & - & - & - & - & - \\
\hline c. $5444+1973 T>C$ & intron 38 & 26514321 & - & - & - & - & - & - & - & - \\
\hline c. $5444+1983 T>C$ & intron 38 & 26514331 & - & - & - & - & - & - & - & - \\
\hline c. $5550+17 \mathrm{~T}>\mathrm{C}$ & intron 38 & 26514536 & - & $\mathrm{T}$ & 0.132 & 0.115 & C & 0.514 & 0.473 & 1.164 \\
\hline c. $5550+141 C>T$ & intron 38 & 26514660 & - & & - & - & & - & - & - \\
\hline BIEC2-226016 & intron 39 & 26516372 & - & $\mathrm{T}$ & 0.309 & 0.308 & G & 0.001 & 0.977 & 1.004 \\
\hline BIEC2-226017 & intron 39 & 26516538 & - & $\mathrm{T}$ & & & C & 0.001 & 0.977 & 1.004 \\
\hline$c .5644 \mathrm{G}>\mathrm{A}$ & exon 40 & 26516727 & p.Ala188 & G & 0.306 & 0.310 & A & 0.017 & 0.895 & 0.980 \\
\hline c. $5926+53 \mathrm{~T}>\mathrm{A}$ & intron 41 & 26519441 & - & $\mathrm{T}$ & 0.493 & 0.489 & A & 0.014 & 0.906 & 1.017 \\
\hline c. $5926+270 T>C$ & intron 41 & 26519658 & - & & - & - & & - & - & - \\
\hline c. $6030+79 G>C$ & intron 42 & 26532238 & - & G & 0.011 & 0.008 & C & - & - & - \\
\hline
\end{tabular}


Table 2 continued

Polymorphisms of the equine DNAH3 gene and association data

\begin{tabular}{|c|c|c|c|c|c|c|c|c|c|c|}
\hline SNP & $\begin{array}{l}\text { DNAH3 } \\
\text { Position }\end{array}$ & $\begin{array}{l}\text { ECA } 13 \\
\text { Position }\end{array}$ & Function & $\begin{array}{c}\text { Allele } \\
1\end{array}$ & $\begin{array}{c}\text { Frequenc } \\
\text { Cases } \\
(\mathrm{n}=230)\end{array}$ & $\begin{array}{l}\text { y Allele } 1 \\
\text { Controls } \\
(n=192)\end{array}$ & $\begin{array}{c}\text { Allele } \\
2\end{array}$ & $\begin{array}{l}\text { Chi- } \\
\text { square }\end{array}$ & $P$-value & $\begin{array}{l}\text { Odds } \\
\text { Ratio }\end{array}$ \\
\hline c. $6120 \mathrm{~T}>\mathrm{C}$ & exon 43 & 26534479 & $\mathrm{p} .=$ & $\mathrm{T}$ & 0.298 & 0.310 & C & 0.149 & 0.700 & 0.943 \\
\hline c. $6505+25 T>G$ & intron 44 & 26536729 & - & G & 0.454 & 0.442 & $\mathrm{~T}$ & 0.126 & 0.723 & 1.051 \\
\hline c. $6786+39 A>G$ & intron 46 & 26542273 & - & & - & - & & - & - & - \\
\hline c.7069A $>\mathrm{G}$ & exon 48 & 26543688 & p.Met2357Val & $G$ & 0.135 & 0.115 & A & 0.729 & 0.393 & 1.197 \\
\hline c.7994A>G & exon 51 & 26551513 & p.GIn2665Arg & G & 0.100 & 0.096 & A & 0.031 & 0.859 & 1.042 \\
\hline c. $8362+2699 \mathrm{G}>\mathrm{A}$ & intron 52 & 26559821 & - & G & 0.093 & 0.083 & A & 0.266 & 0.606 & 1.134 \\
\hline c. $9098 \mathrm{C}>\mathrm{G}$ & exon 53 & 26561025 & p.Ser3033Cys & G & 0.092 & 0.079 & $\mathrm{C}$ & 0.430 & 0.512 & 1.178 \\
\hline c. $10245 G>A$ & exon 53 & 26562172 & $\mathrm{p} .=$ & G & 0.093 & 0.083 & A & 0.266 & 0.606 & 1.134 \\
\hline c. $11247+235 \mathrm{~A}>\mathrm{G}$ & intron 57 & 26576428 & - & A & 0.300 & 0.304 & G & 0.017 & 0.898 & 0.981 \\
\hline c. $11558+725 T>C$ & intron 59 & 26579915 & - & $\mathrm{T}$ & 0.222 & 0.208 & C & 0.222 & 0.637 & 1.083 \\
\hline
\end{tabular}

\begin{tabular}{|c|c|c|c|c|c|c|c|}
\hline Species & p.K345E & p.V511I & p.Q1227R & p.A1882T & p.M2357V & $p \cdot Q 2665 R$ & p.S3033C \\
\hline Horse wildtype & ELLKGKL & FTAVDQQ & ASMQKVI & IHLAFMM & TVVMEYY & AVMQVEL & DSFSIDN \\
\hline Horse mutated & ...E... & ...I. & ...R. & ...т. & $\ldots \mathrm{v}$ & . . R. . & $\ldots c$ \\
\hline Human & .L. & $\ldots$ AAAD & . RE. & s. & 1. & R. . & \\
\hline Mouse & D.. & SFIFLYL & . RQ. . & s. & & & \\
\hline Dog &.$G$ & & $\ldots R E$. & v...s. & $\theta$ & . & \\
\hline Platypus & ...AST & --- & E. IRH. . & v...vs. &.$T$ & V...K. . & \\
\hline Chicken & . ICG. & --- & . VRQ.L & IH.TYSL & .A. & .E. & \\
\hline Fish & S. . SAD. & --- & M.IRA. . & $\ldots$. YSL & .E. DF. & $\ldots Q$. & .L.T. \\
\hline
\end{tabular}

Figure 1

Multiple species alignment of the DNAH3 protein sequence at the sites of non-synonymous variants in the horse. Four of the seven variants lead to amino acid exchanges in highly conserved positions.

Table 3

Protein domain assignment of non-synonymous variants

\begin{tabular}{|c|c|c|c|c|}
\hline Variants & $\begin{array}{l}\text { Corresponding } \\
\text { human position }\end{array}$ & Domain & Remark & Region \\
\hline p.Lys345Glu & 375 & Stem & & $1 . .1390$ \\
\hline p.GIn1227Arg & 1259 & Stem & & $1 . .1390$ \\
\hline p.Ala1882Thr & 1914 & -- & $\begin{array}{c}\text { Between the AAA } 2 \text { and AAA } 3 \\
\text { domains }\end{array}$ & $1904 . .2035$ \\
\hline p.Met2357Val & 2389 & -- & $\begin{array}{c}\text { Between the AAA } 3 \text { and AAA } 4 \\
\text { domains }\end{array}$ & $2285 . .2394$ \\
\hline p.GIn2665Arg & 2697 & Stalk & & $2661 . .2960$ \\
\hline p.Ser3033Cys & 3065 & AAA 5 & & $3045 . .3275$ \\
\hline
\end{tabular}

The numbering refers to the equine protein accession XP_001491903.2. The predicted equine protein differs at its N-terminus from the human protein, possibly due to an annotation error in the horse genome reference sequence. The predicted equine protein shows high homology to its human ortholog starting from amino acid 11 in horse and amino acid 41 in human. The domain positions correspond to the human DNAH3 protein from the UniProtKB/Swiss-Prot accession Q8TD57. 
In previous association studies for RAO on ECA 13, we found the strongest association for the marker BIEC2-224511 (Shakhsi-Niaei et al. 2012). This marker has a raw $P$-value of 0.015 in the 422 horses of this study and thus shows an almost four-fold stronger association than the best-associated marker from the DNAH3 gene. BIEC2-224511 is located at position 24309405 , approximately $2 \mathrm{Mb}$ away from the DNAH3 gene. The best associated marker in the DNAH3 gene (c.-263C >T) is at the left boundary of the DNAH3 gene and thus also physically closest to BIEC2-224511 of all tested markers (Table 2).

The lack of association of the tested DNAH3 markers indicates that variants at the DNAH3 gene are most likely not responsible for the RAO QTL in European Warmblood horses.

\section{Acknowledgements}

The authors would like to thank Vidhya Jagannathan for help with dbSNP submission and Brigitta Colomb for expert technical assistance. This study was supported in part by grants from the Swiss National Science Foundation (310000-116502) and by a fellowship from the Iranian Ministry of Science, Research and Technology to M. S.-N.

\section{References}

Afzelius BA (1976) A Human Syndrome Caused by Immotile Cilia. Science 193, 317-319

Bartoloni L, Blouin JL, Maiti AK, Sainsbury A, Rossier C, Gehrig C, She JX, Marron MP, Lander ES, Meeks M, Chung E, Armengot M, Jorissen M, Scott HS, Delozier-Blanchet CD, Gardiner RM, Antonarakis SE (2001) Axonemal Beta Heavy Chain Dynein DNAH9: cDNA Sequence, Genomic Structure, and Investigation of Its Role in Primary Ciliary Dyskinesia. Genomics 72, 21-33

Brokaw CJ (2009) Thinking about flagellar oscillation. Cell Motil Cytoskeleton 66, 425-436

Cunningham FM, Dunkel B (2008) Equine recurrent airway obstruction and insect bite hypersensitivity: Understanding the diseases and uncovering possible new therapeutic approaches. Vet J 177, 334-344

Deaton CM (2006) The role of oxidative stress in an equine model of human asthma. Redox Rep 11, 46-52

DiBella LM, King SM (2001) Dynein motors of the Chlamydomonas flagellum. Int Rev Cytol 210, 227-268

Gerber V, Baleri D, Klukowska-Rötzler J, Swinburne JE, Dolf G (2009) Mixed Inheritance of Equine Recurrent Airway Obstruction. J Vet Intern Med 23, 626-630

Hayashi S, Shingyoji C (2008) Mechanism of flagellar oscillation - bending-induced switching of dynein activity in elastase-treated axonemes of sea urchin sperm. J Cell Sci 121, 2833-2843

Holzbaur ELF, Vallee RB (1994) DYNEINS: Molecular Structure and Cellular Function. Annu Rev Cell Biol 10, 339-372

Knowles MR, Boucher RC (2002) Mucus clearance as a primary innate defense mechanism for mammalian airways. J Clin Invest 109, 571-577

Laumen E, Doherr MG, Gerber V (2010) Relationship of horse owner assessed respiratory signs index to characteristics of recurrent airway obstruction in two Warmblood families. Equine Vet J 42, 142-148

Leclere M, Lavoie-Lamoureux A, Lavoie JP (2011) Heaves, an asthma-like disease of horses. Respirology 16, 1027-1046

Léguillette R (2003) Recurrent airway obstruction-heaves. Vet Clin Equine 19, 63-86

Lee L (2011) Mechanisms of mammalian ciliary motility: Insights from primary ciliary dyskinesia genetics. Gene 473, 57-66

Maiti AK, Mattéi MG, Jorissen M, Volz A, Zeigler A, Bouvagnet P (2000) Identification, tissue specific expression, and chromosomal localisation of several human dynein heavy chain genes. Eur J Hum Genet 8, 923-932 
Purcell S, Neale B, Todd-Brown K, Thomas L, Ferreira MAR, Bender D, Maller J, Sklar P, de Bakker PIW, Daly MJ, Sham PC (2007) PLINK: A Tool Set for Whole-Genome Association and Population-Based Linkage Analyses. Am J Hum Genet 81, 559-575

Ramseyer A, Gaillard C, Burger D, Straub R, Jost U, Boog C, Marti E, Gerber V (2007) Effects of Genetic and Environmental Factors on Chronic Lower Airway Disease in Horses. J Vet Intern Med 21, 149-156

Rossman CM, Forrest JB, Ruffin RE, Newhouse MT (1980) Immotile cilia syndrome in persons with and without Kartagener's syndrome. Am Rev Respir Dis 121, 1011-1016

Shakhsi-Niaei M, Klukowska-Rötzler J, Drögemüller C, Swinburne J, Ehrmann C, Saftic D, Ramseyer A, Gerber V, Dolf G, Leeb T (2012) Replication and fine-mapping of a QTL for recurrent airway obstruction in European Warmblood horses. Anim Genet 43, 627-631

Swinburne JE, Bogle H, Klukowska-Rötzler J, Drögemüller M, Leeb T, Temperton E, Dolf G, Gerber V (2009) A whole-genome scan for recurrent airway obstruction in Warmblood sport horses indicates two positional candidate regions. Mamm Genome 20, 504-515 